Technical note

\title{
First-in-human imaging using a MR-compatible e4D ultrasound probe for motion management of radiotherapy
}

\author{
Bryan P. Bednarz ${ }^{\mathrm{a}, *}$, Sydney Jupitz ${ }^{\mathrm{a}}$, Warren Lee ${ }^{\mathrm{b}}$, David Mills ${ }^{\mathrm{b}}$, Heather Chan ${ }^{\mathrm{b}}$, \\ Timothy Fiorillo $^{\mathrm{b}}$, James Sabitini ${ }^{\mathrm{b}}$, David Shoudy ${ }^{\mathrm{b}}$, Aqsa Patel ${ }^{\mathrm{b}}$, Jhimli Mitra ${ }^{\mathrm{b}}$, \\ Shourya Sarcar ${ }^{\mathrm{b}}$, Bo Wang ${ }^{\mathrm{b}}$, Andrew Shepard ${ }^{\mathrm{a}, \mathrm{c}}$, Charles Matrosic ${ }^{\mathrm{a}}$, , James Holmes ${ }^{\mathrm{e}}$, \\ Wesley Culberson ${ }^{\mathrm{a}}$, Michael Bassetti ${ }^{\mathrm{f}}$, Patrick Hill ${ }^{\mathrm{f}}$, Alan McMillan ${ }^{\mathrm{e}}$, James Zagzebski ${ }^{\mathrm{a}}$, \\ L. Scott Smith ${ }^{\mathrm{b}}$, Thomas K. Foo ${ }^{\mathrm{b}}$ \\ ${ }^{a}$ Medical Physics, University of Wisconsin-Madison, Madison, WI 53705, United States \\ ${ }^{\mathrm{b}}$ GE Global Research, Niskayuna, NY 12309, United States \\ ${ }^{\mathrm{c}}$ Department of Radiation Oncology, University of Iowa, Iowa City, IA 52242, United States \\ ${ }^{\mathrm{d}}$ Department of Radiation Oncology, University of Michigan, Ann Arbor, MI 48109, United States \\ ${ }^{\mathrm{e}}$ Department of Radiology, University of Wisconsin-Madison, Madison, WI 53705, United States \\ ${ }^{\mathrm{f}}$ Department of Human Oncology, University of Wisconsin-Madison, Madison, WI 53705, United States
}

\section{A R T I C L E I N F O}

\section{Keywords:}

Ultrasound

Motion management

Radiotherapy

\begin{abstract}
A B S T R A C T
Purpose: Respiration-induced tumor or organ positional changes can impact the accuracy of external beam radiotherapy. Motion management strategies are used to account for these changes during treatment. The authors report on the development, testing, and first-in-human evaluation of an electronic 4D (e4D) MR-compatible ultrasound probe that was designed for hands-free operation in a MR and linear accelerator (LINAC) environment.

Methods: Ultrasound components were evaluated for MR compatibility. Electromagnetic interference (EMI) shielding was used to enclose the entire probe and a factory-fabricated cable shielded with copper braids was integrated into the probe. A series of simultaneous ultrasound and MR scans were acquired and analyzed in five healthy volunteers.

Results: The ultrasound probe led to minor susceptibility artifacts in the MR images immediately proximal to the ultrasound probe at a depth of $<10 \mathrm{~mm}$. Ultrasound and MR-based motion traces that were derived by tracking the salient motion of endogenous target structures in the superior-inferior (SI) direction demonstrated good concordance (Pearson correlation coefficients of 0.95-0.98) between the ultrasound and MRI datasets.

Conclusion: We have demonstrated that our hands-free, e4D probe can acquire ultrasound images during a MR acquisition at frame rates of approximately 4 frames per second (fps) without impacting either the MR or ultrasound image quality. This use of this technology for interventional procedures (e.g. biopsies and drug delivery) and motion compensation during imaging are also being explored.
\end{abstract}

\section{Introduction}

Clinical outcomes associated with external beam radiotherapy depend on the precise delivery of a planned amount of absorbed dose to the tumor while avoiding healthy sensitive structures or organs. Respiration-induced tumor or organ positional changes can decrease the precision of each treatment session, expanding the treatment region and exposing a greater volume of healthy tissue to radiation damage.
Thoracic tumor displacements of more than $20 \mathrm{~mm}$ have been observed during normal breathing [1] whereas abdominal tumors close to the diaphragm such as tumors in the pancreas and kidneys can move more than $20 \mathrm{~mm}$ and tumors in the liver can move more than $30 \mathrm{~mm}[2,3]$. Treatment margins are often expanded to account for tumor positional variability and uncertainty. However, these expansions inevitably come at the cost of increased dose to normal tissue leading to sub-optimal treatment outcomes.

\footnotetext{
* Corresponding author.

E-mail address: bbednarz2@wisc.edu (B.P. Bednarz).
} 
Motion management strategies have been used during treatment delivery to reduce motion encompassing margins $[4,5]$. These include tumor motion control by forced shallow breathing with abdominal compression [6,7], breath-hold [8,9], and respiratory gating using either external respiration signals [10] or artificially implanted metallic fiducial markers [11]. While each of these techniques have been used clinically with varying degrees of success, significant quality assurance and reproducibility challenges associated with all of these techniques have been documented $[12,13]$. Real-time tracking strategies have also been introduced that dynamically reposition the radiation beam in order to follow the motion of the tumor [14-16]. Tumor motion is often determined by real-time imaging of the tumor itself using fluoroscopy, ultrasound or magnetic resonance imaging (MRI) or by using artificially implanted fiducial markers such as gold with $\mathrm{kV}$ and/or MV imaging [17], radioactive markers [18], and electromagnetic transponders [19]. In the case of implanted transponders, migration and stability during respiratory gating limit the accuracy of this technique [13,20,21]. Furthermore, the poor health of many patients prevents them from being candidates for implantation or puts them at serious risk of developing complications following surgery [22,23]. In addition, the position of the implanted markers change as the tumor shrinks in response to radiation therapy, requiring re-implantation. Also, there is a serious concern about concomitant radiation dose received from fluoroscopy-guided real-time tracking [24,25].

Ultrasound is an alternative non-invasive real-time strategy that does not expose the patient to extra radiation. The first application of ultrasound in radiotherapy was to monitor interfractional motion between treatments [26]. Multiple commercial systems were developed to improve patient positioning prior to the beam delivery including the BMode Acquisition and Targeting (BAT) system (NOMOS Corp., Cranberry Township, PA), the SonArray system (Varian Medical Systems, Palo Alto, CA), and the Clarity system (Elekta AB, Stockholm, Sweden). However, these systems shared multiple shortcomings that limited their use for intrafraction motion management including probe-induced anatomical deformations [27-29], quality and consistency of freehand ultrasound-based patient alignment [30,31], and lack of real-time monitoring during beam delivery [32]. Several research groups are investigating the use of static hardware fixtures or robotic devices [28,33-36] along with advanced estimation techniques [37] to address these limitations. Comprehensive reviews on ultrasound-guided radiotherapy can be found elsewhere [32,38,39].

More recently, MRI-guided radiotherapy has emerged as a promising image guidance strategy providing excellent soft tissue contrast and real-time imaging capabilities [40]. Most patients are treated using beam gating with coached breath holds [41], but real-time tumor tracking techniques are also being evaluated. In a recent article published in this journal it was argued that combination MRI and linear accelerator (MR-LINAC) systems will replace conventional imageguided radiotherapy (IGRT) systems within 15 years [42]. Be that as it may, it is clear that widespread use of this technology will be encumbered by unresolved technical challenges and the large capital investment required to purchase, install and operate this technology $[40,42]$, which is approximately 3-4 times the cost of an advanced high-end LINAC treatment system.

Therefore, despite this progress, there is currently no de facto standard for motion management in the clinic. There remains a clinical need for widely accessible, real-time, image-guidance technologies that can overcome the technical limitations and reduce the cost associated with current motion management strategies. To this end, our team from University of Wisconsin-Madison and General Electric Research has developed and is evaluating the first-ever MR-compatible e4D ultrasound probe for real-time motion management. The hands-free ultrasound probe has been designed to monitor the motion of tumors or internal motion surrogates in the abdominal region during radiation beam delivery. This note highlights the development, testing, and firstin-human evaluation of the ultrasound probe in an MR environment.

\section{Methods}

\section{A. Ultrasound Probe Development}

A detailed description of the probe development is provided in Lee, et al [43], and an abbreviated summary is discussed here. A low-profile ultrasound probe was designed to permit hands-free operation for imaging applications in the abdominal region. A matrix array transducer containing $\sim 18,000$ piezoelectric elements with an active aperture of $46.8 \mathrm{~mm} \times 21.5 \mathrm{~mm}$ was constructed. This transducer also contains embedded custom beamforming ASICs to reduce the number of independent receive signals to match the channels of the GE Vivid E95 ultrasound system. An FPGA board located inside the probe communicates with the ultrasound system and configures the internal beamforming ASIC. The acoustic stack consists of an interface layer, a single crystal PMN-PT piezoelectric layer, a filled graphite inner matching layer and a polymeric outer matching layer. The active aperture was composed of three identical modules which were tiled side by side to form the wide aperture. The modules were designed to allow the elements to remain on a consistent pitch even across module boundaries. An illustration of the probe and a description of the internal components is shown in Fig. 1. Initial acoustic characterization of the probe operating at $3.5 \mathrm{MHz}$ center frequency showed an $82 \%-6 \mathrm{~dB}$ fractional bandwidth and an excellent element yield of greater than $99 \%$. The maximum penetration is $18 \mathrm{~cm}$ with a $2 \mathrm{~mm}$ lateral resolution at $7 \mathrm{~cm}$ depth. In 3D mode at a depth of $18 \mathrm{~cm}$, the volume frame rate was 4 frames per second, for the large volume field-of-view (FOV) of 40 degrees $\times 28.6$ degrees. It is noted that the acquisition frame rate is inversely proportional to the imaging FOV, and higher frame rates can be achieved for a more directed narrower FOV or reduced imaging depth.

Ultrasound probe safety testing was completed prior to scanning in humans in accordance with U.S. FDA MRI and ultrasound nonsignificant risk guidelines IEC60601-2-33 and IEC60601-2-37 standards. Safety tests included: (1) measurements of the acoustic pressure at different imaging modes, (2) measurements of electrical leakage and absence of high voltage breakdown, (3) thermal management (temperature of probe and cable), (4) eddy-current induced temperature rise of probe surface as well as probe cable during MR scanning using a fast spin echo stress pulse sequence. The test results were reviewed by the GE environmental, health, and safety officers and the University of Wisconsin IRB to certify the probe for human subject testing.

\section{B. MR compatibility}

In MR images, paramagnetic and ferromagnetic materials can distort the homogeneity of the $\mathrm{B}_{0}$ magnetic field and cause major susceptibility artifacts that appear as signal loss or geometric distortions. In order to evaluate the MR-compatibility of the ultrasound probe materials, all suspect components were individually evaluated in an MR system (3 T GE MR750, GE Healthcare, Waukesha, WI) and corresponding susceptibility artifacts in the acquired images were quantified. Components that led to significant artifacts were either replaced by less magnetic parts or removed entirely. Lee et al [43] demonstrated that these changes had minimal impact on image quality.

Electromagnetic interference (EMI) can severely degrade both ultrasound and MR image quality. EMI shielding was designed for both the probe and cable to significantly reduce this interference. EMI can also impact ultrasound image quality during LINAC beam delivery [33,36]. A 3D printed aluminum EMI shield was used to enclose the entire probe except the active acoustic aperture. The active acoustic aperture was shielded with a thin $0.012 \mathrm{~mm}$ layer of aluminum. This shield also protected the probe electronics from fast gradient field switching (dB) $\mathrm{dt}$ ) during MR acquisition, as well as high-frequency radiofrequency (RF) field effects. In addition, a factory-fabricated 8.5-meter-long cable, shielded with multiple copper braids, transmits signals and commands between the probe located in the bore of the MRI system and the ultrasound system located outside the MRI scan room in the control room. A custom-made insert coupled the cable shield to the grounded waveguide where the cable exits the MRI shielded room and prevented RF 


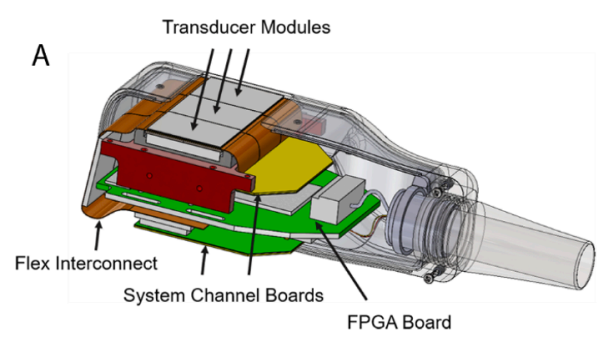

B

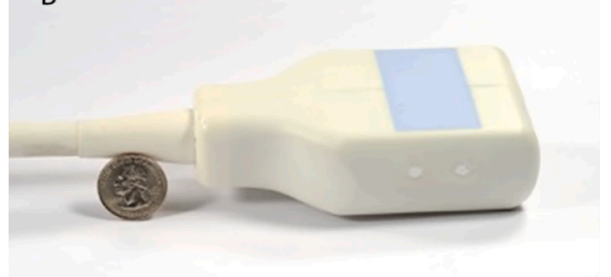

Fig. 1. Schematic diagram of the MR-compatible ultrasound probe with various probe components labeled (left). The arrangement of the electronics within the probe housing was optimized to reduce $B_{0}$ susceptibility artifacts in MR images while maintaining a low-profile form factor to allow hands-free optimization. A picture of the first prototype is shown with a quarter for scale (right).

interference.

C. First-in-human Imaging

In order to evaluate the performance of the ultrasound probe in an MR environment, a series of simultaneous ultrasound and MR scans were conducted in five healthy volunteers. All scans were performed as part of an IRB-approved protocol, with written informed consent received from each subject. During image acquisition, the ultrasound probe was placed either in an inter- or sub-costal position to view liver vasculature and a nylon strap was utilized for probe stabilization. 4D ultrasound images were acquired in harmonic imaging mode (1.7/3.3 $\mathrm{MHz}$ ) on the GE Vivid E95 system located in the MR control room. A schematic of the image acquisition setup is provided in Fig. 2. The ultrasound probe was easily placed below the 30-channel array AIR anterior receive coil. MRI was performed using the anterior array in combination with the posterior array receive coil on a GE 3 T Premier scanner (GE Healthcare, Waukesha, WI). Dynamic 2D MR images were simultaneously acquired using a fast spoiled gradient echo (FSPGR) multi-phase acquisition with temporal resolution of $3 \mathrm{fps}$ (TE/TR $=1.1$ $\mathrm{ms} ; \mathrm{FOV}=40 \mathrm{~cm} / 32 \mathrm{~cm}$; acquired matrix $=256 \times 192$, ASSET $=2)$ in a GE 3 T MR750 MRI scanner. Specific to tracking motion, 6-8 contiguous two-dimensional sagittal slices were acquired for each volunteer simultaneous with the 4D ultrasound acquisition. Sagittal slices were used to capture the respiratory induced motion of vessels that move predominantly in the superior-inferior (SI) direction in the liver. During acquisition, both MR and ultrasound datasets were streamed to an external workstation that was configured with an 8-core, $3.2 \mathrm{GHz}$ Intel

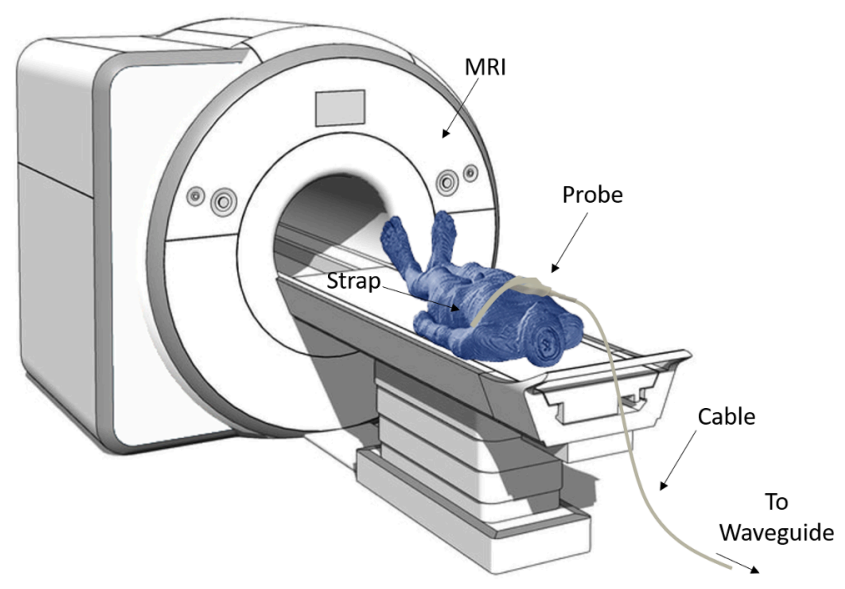

Fig. 2. A schematic of the simultaneous US/MR image acquisitions. A nylon strap stabilizes the probe to the patient's surface. The probe is attached to the strap using a swivel so that the strap position does not impact the orientation of the probe. A flexible surface coil is placed over the probe (not shown) The cable is draped over the shoulder of the volunteer and is passed through the waveguide connecting the image room to the control room where the GE Vivid V95 system resides.
Xeon processor, 512 GB of RAM, and an NVIDIA GeForce GTX Titan X graphics processing unit (GPU). The FSGRE pulse sequence was modified to transmit a TTL logic signal via a PCI interface at the start and end of data acquisition at each sagittal slice location to synchronize the timestamps between the MR and US images. During MR acquisitions, as 3D ultrasound data streams into the workstation continuously, MR DICOM packets structured as DICOM files are streamed into the workstation via a socket connection without using the DICOM network transfer protocol. The kernel driver on the workstation has been modified to intercept the TTL signal pulse and establish the logical boundaries of the US volumes and the MR DICOM packets. This allowed direct indexing in time between the MR and US volumes. The US volumes are saved in beam-space (i.e., not converted into physical $\mathrm{x}, \mathrm{y}, \mathrm{z}$ coordinates) and time-stamped only during simultaneous MR-US acquisition. Each series of the MR acquisition (i.e. 6-8 slices encompassing the US FOV) is comprised of 200 images (240 3D U/S volumes) at the same slice location, allowing the capture of several respiratory cycles at each location.

During each healthy volunteer imaging session, the probe sustained internal heating. Initial heating was mainly due to the heat emanating from the healthy volunteer and additional heating was observed during active $4 \mathrm{D}$ ultrasound acquisition. If the temperature exceeded the internal safety setting, a few millidegrees below the $43^{\circ} \mathrm{C}$ regulatory limit, the imager automatically stopped transmitting and temporarily halted ultrasound acquisition. It was observed that by reducing the acoustic power, the heating rate during acquisition could be reduced. Consequently, a protocol was followed where the probe was placed on the volunteer using peak acoustic power, then once the target vessel was identified the acoustic power was reduced. The reduction in acoustic power was optimized such that only the imaging at depths beyond the location of the target vessel was impacted. While the posterior boundary of the liver, kidneys, and vertebrae are helpful for image registration, they are not integral for this application. These features are still visible with reduced acoustic power, but the contrast is reduced.

Using five volunteer datasets, salient vessels were tracked retrospectively in both the 4D ultrasound and 2D FSPGR scan. The tracking was performed in 3D on the ultrasound data and in 2D on the MR data using an advanced block matching approach, which has been described previously [44]. Initialization of the tracking algorithm requires a manual first frame contour be drawn by the user. For each subsequent image frame in the dataset, the tracking algorithm determines the new contour of the vessel from which the centroid of the vessel is calculated. The centroid position of the vessel tracked in each frame shows the motion of the vessel over time. The phase correlation of the vessel motion in both image sets during respiration was determined using a Pearson product moment correlation coefficient (CC). The CC is indicative of the degree of phase correlation between two motion vectors and has been used extensively to show motion correlation [45-47].

In addition to the FSPGR scan, a 3D T1-weighted FLEX (water-only) fast spin echo scan was acquired at the end of expiration for each volunteer. This scan provided a 3D MR dataset in the MR reference 
frame to which the 3D ultrasound dataset could be registered, as opposed to trying to register 3D ultrasound directly to 2D MR. The superficial field distortions in the 3D T1-weighted FLEX scan helped to localize the ultrasound probe in the MR volume. An end expiration volume in the $4 \mathrm{D}$ ultrasound dataset was manually registered to the $3 \mathrm{D}$ MR dataset using a rigid registration of multiple salient anatomical landmarks in both datasets. The resulting transformation matrix was then applied to the ultrasound vessel tracked centroids transforming the tracked ultrasound vessel centroids, and therefore motion, into the MR the coordinate system. Therefore, the tracking results of the 4D ultrasound and dynamic 2D MR scans were temporally and spatially registered. The SI motion could then be identified, extracted, and compared for both datasets.

\section{Results}

Representative human images of the liver acquired with the ultrasound probe during simultaneous MRI scans are shown in Fig. 2. For these volunteer scans, the probe was tactically placed by the sonographer in an intercostal position on the volunteer in order to capture the motion of salient features including a tracking vessel (TV) and to minimize effects of acoustic shadowing caused by the vertebrae (Vt) on the registration process. This positioning also helped to stabilize the probe and reduce probe motion due to respiration. Excellent registration was achieved between the 4D ultrasound scans, and the 3D T1-weighted flex (water-only) scans (Fig. 2A-F) and the 2D FSPGR scans (not shown) using a rigid landmark-based approach for all five subjects. Scan sessions
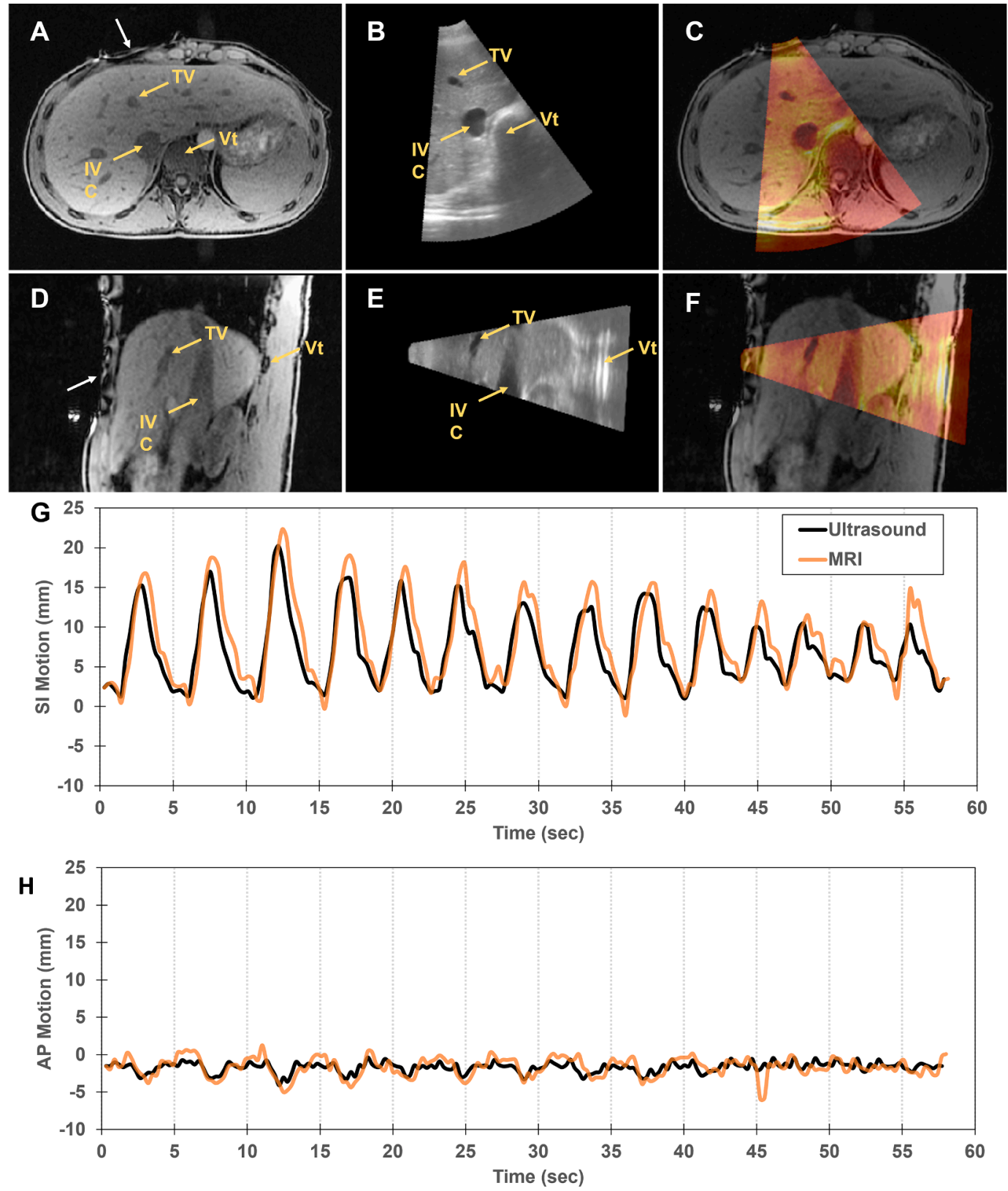

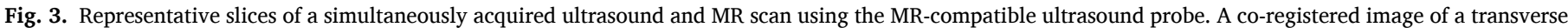

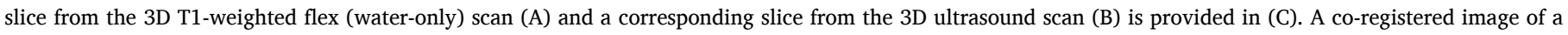

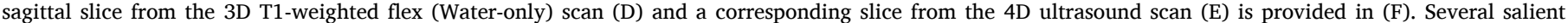

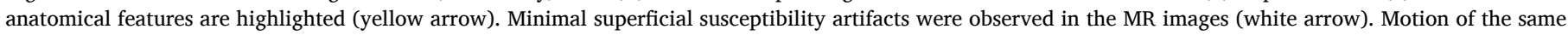

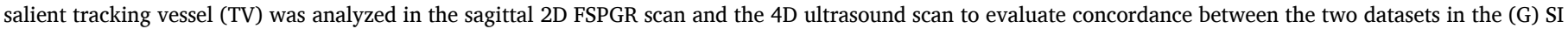
and $(\mathrm{H})$ AP direction. (For interpretation of the references to colour in this figure legend, the reader is referred to the web version of this article.) 
for the 5 volunteers lasted approximately one hour on average which included the localizer scan, the 3D T1-weighted flex (water-only) scan and the multi-slice (up to eight) FSPGR scans.

The ultrasound probe led to small superficial susceptibility artifacts in the MR images (Fig. 3A) that did not extend far from the surface. These artifacts were mostly visible in subcutaneous fat and abdominal muscle $<2 \mathrm{~cm}$ from the surface of the volunteer. No additional MRI image artifacts due to the electronic operation of the ultrasound probe were observed. Ultrasound image artifacts due to MR gradient switching were also not observed. Ultrasound and MR-based motion traces derived from tracking a salient TV in the superior-inferior (SI) and anteriorposterior (AP) direction are provided in Fig. 3G\&H to demonstrate concordance between the ultrasound and MRI datasets. The Pearson correlation coefficients in the SI and AP direction between the tracked datasets in this subject (i.e. Subject 1) along with four additional volunteer datasets are provided in Table 1 . Also included in the table are values for the mean and maximum left-right (LR) motion derived from the ultrasound dataset and mean and maximum amplitude differences (i. e. peaks) between tracked ultrasound and MR positions in the SI direction. The average correlation coefficient in the SI and AP direction for all datasets considered was 0.96 and 0.71 , respectively. The lower correlation in the AP direction is predominately due to the limited range of vessel motion in this direction. For example, the position of tracked centroids in Subject 1 in both the ultrasound and MR images were less than $\pm 2 \mathrm{~mm}$ for $65 \%$ of the $60 \mathrm{~s}$ breathing trace, which is less than three pixels (i.e., $1.5625 \mathrm{~mm} / \mathrm{pixel}$ ) in an MR image frame. The range of motion was even smaller during the last $20 \mathrm{~s}$ of the measured breathing trace as shown in Fig. $3 \mathrm{H}$. The maximum amplitude difference in the SI direction over all datasets was $5.63 \mathrm{~mm}$ in Subject 1. This large difference is due to a tracking error that occurred during analysis in the MR dataset. The tracking algorithm did a poor job tracking the vessel in this frame, which was due to the tracked vessel disappearing in this frame due to loss of blood contrast. This also led to larger tracking errors in subsequent frames before the tracking algorithm was able to adjust back to the correct position. It is worth noting that only 3 out of 1000 frames $(0.3 \%)$ analyzed had an amplitude difference greater than $5 \mathrm{~mm}$. The American Association of Physicists in Medicine recommends for external beam radiotherapy the use of motion management when the target motion is greater than $5 \mathrm{~mm}$ [5]. Therefore, errors in target tracking should also not exceed $5 \mathrm{~mm}$.

\section{Discussion and conclusions}

Our team has developed an MR-compatible, e4D ultrasound probe for motion management of radiotherapy. The probe is low-profile in design and permits hands-free operation after initial placement. To date, a total of five healthy volunteers have been scanned to evaluate the performance of the ultrasound probe during MRI acquisition. The minimal susceptibility artifacts that were detected superficially in images of the volunteers did not inhibit analysis or impact overall image quality. None of the healthy volunteers complained of discomfort associated with the probe during set-up and acquisition. Visual inspection did not yield evidence of positional drift of the probe, implying the position of the ultrasound is stable over the course of imaging. Since mechanical contact and fixed alignment between the probe and patient are key in creating robust, high quality images, we visually inspected the probe for evidence of both translation and rotation during scans. All indications are that the position of the ultrasound probe remained stable during the imaging session. Further improvement in probe localization and repositioning is an active area of research. In addition, the five volunteer datasets in this paper captured sufficient anatomical features that allowed for a rigid landmark-based registration technique to be employed. This image registration technique may not be appropriate for all datasets as a variety of probe positions and liver regions were interrogated. Future work also includes the development of a robust registration technique to cover different imaging scenarios.

Initially, when the probe was covered with blankets or sheets and during longer scan sessions the probe temperature sometimes rose significantly. However, we found that heating could be mitigated by removing the overlaying covers and improving free air convection, lowering the acoustic power, optimizing the time when the probe is actively transmitting, and allowing additional cooling time between MR slice acquisitions. Reducing the acoustic power was effective at reducing the heating rate and did not impact the integrity of the ultrasound imaging within the region of interest. Additional cooling techniques are also under investigation.

The intended use of the probe is to aid in motion management for radiotherapy of abdominal cancers. In our approach, simultaneous ultrasound and MR images will be acquired prior to treatment delivery. The MR images will provide excellent soft tissue contrast of the moving and potentially deforming tumor volume and surrounding healthy tissue. The use of simultaneously acquired dynamic 4D ultrasound will be two-fold. First, ultrasound images will be used to help retrospectively stack dynamic 2D MR slices into appropriate respiratory phases to reconstruct 4D MR volumes. 3D MRI is currently not ideal for real-time imaging respiration-induced motion since it suffers from low temporal and/or spatial resolution when large volumes are scanned. The stack of 2D MR images can be resorted and reformatted into 3D volumes that represent each respiratory phase. Prior work has used retrospective binning approaches to reconstruct 4D MR using external surrogates such as belt respirations sensors [48] or internal surrogates such as body area $[49,50]$, mutual information [51], manifold learning [52], and deformation fields of interleaved navigator slices [53]. More recently, internal surrogates captured by ultrasound imaging have also been used for retrospective binning [54]. Second, with a matching probe position, ultrasound scans will be acquired in real-time during treatment and rapidly matched to the pre-treatment ultrasound and MR volumes. The ultrasound probe will image the tumor motion or an internal motion surrogate during treatment thereby allowing for tracking of the target position from the 4D ultrasound images. The positional changes will determine the different respiratory states that will be matched to the pre-treatment/planning calibration image set consisting of a simultaneous 4D ultrasound and 2D MRI acquisition that will supply matched pairs or sets of images where each time-point or phase represents a specific respiratory state. The ultrasound image sets will be rapidly matched and salient features within the images will be tracked using the aforementioned block-matching algorithm $[44,55]$. As each respiratory phase determined from ultrasound has a temporally matched MR image,

\section{Table 1}

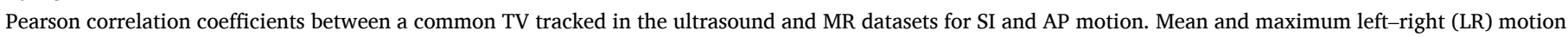

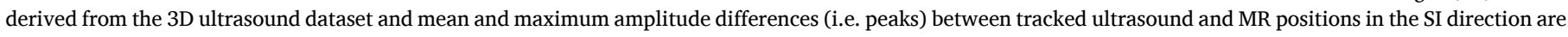
also provided.

\begin{tabular}{|c|c|c|c|c|c|c|}
\hline Subject \# & SI Correlation & AP Correlation & Mean LR Amplitude (mm) & Max LR Amplitude (mm) & Mean Diff in SI Amplitude (mm) & Max Diff in SI Amplitude (mm) \\
\hline 1 & 0.97 & 0.64 & 1.19 & 3.76 & 2.48 & 5.63 \\
\hline 2 & 0.98 & 0.77 & 1.65 & 3.71 & 1.25 & 3.34 \\
\hline 3 & 0.95 & 0.77 & 2.67 & 8.50 & 1.48 & 3.25 \\
\hline 4 & 0.95 & 0.68 & 1.17 & 3.99 & 1.84 & 3.47 \\
\hline 5 & 0.95 & 0.67 & 1.48 & 5.39 & 0.93 & 2.65 \\
\hline Average & 0.96 & 0.71 & 1.63 & 5.07 & 1.60 & 3.67 \\
\hline
\end{tabular}


the MR images at each respiratory phase can be displayed, providing a virtual real-time 2D or 3D MR image of the respiratory motion of the patient in a standard LINAC system. Notably, current commercial MRLINAC clinical protocols only permit 2D acquisitions for real-time imaging $[56,57]$. Preliminary dosimetry data acquired in a deformable abdominal phantom has demonstrated that the ultrasound probe can accurately control beam gating during a liver stereotactic body radiotherapy (SBRT) treatment [58].

The first human images presented herein demonstrate the potential of our multi-modal imaging approach. Our hands-free, e4D probe can acquire ultrasound images during a MR acquisition without impacting the quality of either the MR or ultrasound images. While the initial use of this technology was intended for radiotherapy, applications related to interventional procedures (e.g. biopsies and drug delivery) and motion compensation during imaging are also being explored.

\section{Acknowledgements}

The authors would like to thank Carl Chalek and Robert Darrow for their contributions to system integration.

Research reported in this publication was supported by the National Cancer Institute of the National Institutes of Health under Award Number R01CA190298. The content is solely the responsibility of the authors and does not necessarily represent the official views of the National Institutes of Health.

\section{References}

[1] Seppenwoolde Y, Shirato H, Kitamura K, Shimizu S, Van Herk M, Lebesque JV et al. Precise and real-time measurement of 3D tumor motion in lung due to breathing and heartbeat, measured during radiotherapy. Int J Radiat Oncol Biol Phys 2002;53:822-34. https://doi.org/10.1016/S0360-3016(02)02803-1.

[2] Langen KM, Jones DTL. Organ motion and its management. Int J Radiat Oncol Biol Phys 2001;50:265-78. https://doi.org/10.1016/S0360-3016(01)01453-5.

[3] Suramo I, Paivansalo M, Myllyla V. Cranio-caudal movements of the liver, pancreas and kidneys in respiration. Acta Radiol - Ser Diagnosis 1984;25:129-31. https:// doi.org/10.1177/028418518402500208.

[4] Bertholet J, Knopf A, Eiben B, McClelland J, Grimwood A, Harris E, et al. Real-time intrafraction motion monitoring in external beam radiotherapy. Phys Med Biol 2019;64:15TR01. https://doi.org/10.1088/1361-6560/ab2ba8.

[5] Keall PJ, Mageras GS, Balter JM, Emery RS, Forster KM, Jiang SB, et al. The management of respiratory motion in radiation oncology report of AAPM Task Group 76. Med Phys 2006;33:3874-900. https://doi.org/10.1118/1.2349696.

[6] Wulf J, Hädinger U, Oppitz U, Olshausen B, Flentje M. Stereotactic radiotherapy of extracranial targets: CT-simulation and accuracy of treatment in the stereotactic body frame. Radiother Oncol 2000;57:225-36. https://doi.org/10.1016/s01678140(00)00226-7.

[7] Lax I, Blomgren H, Näslund I, Svanström R. Stereotactic radiotherapy of malignancies in the abdomen: methodological aspects. Acta Oncol (Madr) 1994;33: 677-83. https://doi.org/10.3109/02841869409121782.

[8] Wong JW, Sharpe MB, Jaffray DA, Kini VR, Robertson JM, Stromberg JS, et al. The use of active breathing control (ABC) to reduce margin for breathing motion. Int J Radiat Oncol Biol Phys 1999;44:911-9. https://doi.org/10.1016/s0360-3016(99) 00056-5.

[9] Mah D, Hanley J, Rosenzweig KE, Yorke E, Braban L, Ling CC, et al. Technical aspects of the deep inspiration breath-hold technique in the treatment of thoracic cancer. Int J Radiat Oncol Biol Phys 2000;48:1175-85. https://doi.org/10.1016/ S0360-3016(00)00747-1.

[10] Berbeco RI, Nishioka S, Shirato H, Jiang SB. Residual motion of lung tumors in endof-inhale respiratory gated radiotherapy based on external surrogates. Med Phys 2006;33:4149-56. https://doi.org/10.1118/1.2358197.

[11] Shimizu S, Shirato H, Ogura S, Akita-Dosaka H, Kitamura K, Nishioka T, et al. Detection of lung tumor movement in real-time tumor-tracking radiotherapy. Int $J$ Radiat Oncol Biol Phys 2001;51:304-10. https://doi.org/10.1016/s0360-3016(01) 01641-8.

[12] Jiang SB, Wolfgang J, Mageras GS. Quality assurance challenges for motionadaptive radiation therapy: gating, breath holding, and four-dimensional computed tomography. Int J Radiat Oncol Biol Phys 2008;71. https://doi.org/ 10.1016/j.ijrobp.2007.07.2386.

[13] Kothary N, Dieterich S, Louie JD, Chang DT, Hofmann LV, Sze DY. Percutaneous implantation of fiducial markers for imaging-guided radiation therapy. Am J Roentgenol 2009;192:1090-6. https://doi.org/10.2214/AJR.08.1399.

[14] Keall PJ, Kini VR, Vedam SS, Mohan R. Motion adaptive x-ray therapy: a feasibility study. Phys Med Biol 2001;46:1-10. https://doi.org/10.1088/0031-9155/46/1/ 301.
[15] Keall PJ, Colvill E, O'Brien R, Ng JA, Poulsen PR, Eade T, et al. The first clinical implementation of electromagnetic transponder-guided MLC tracking. Med Phys 2014;41:020702. https://doi.org/10.1118/1.4862509.

[16] Fast MF, Kamerling CP, Ziegenhein P, Menten MJ, Bedford JL, Nill S, et al. Assessment of MLC tracking performance during hypofractionated prostate radiotherapy using real-time dose reconstruction. Phys Med Biol 2016;61:1546-62. https://doi.org/10.1088/0031-9155/61/4/1546.

[17] Wiersma RD, Mao W, Xing L. Combined $\mathrm{kV}$ and MV imaging for real-time tracking of implanted fiducial markers. Med Phys 2008;35:1191-8. https://doi.org/ 10.1118/1.2842072.

[18] Chamberland M, Wassenaar R, Spencer B, Xu T. Performance evaluation of realtime motion tracking using positron emission fiducial markers. Med Phys 2011;38: 810-9. https://doi.org/10.1118/1.3537206.

[19] Balter JM, Wright JN, Newell LJ, Friemel B, Dimmer S, Cheng Y, et al. Accuracy of a wireless localization system for radiotherapy. Int J Radiat Oncol Biol Phys 2005; 61:933-7. https://doi.org/10.1016/j.ijrobp.2004.11.009.

[20] Delouya G, Carrier JF, Béliveau-Nadeau D, Donath D, Taussky D. Migration of intraprostatic fiducial markers and its influence on the matching quality in external beam radiation therapy for prostate cancer. Radiother Oncol 2010;96:43-7. https://doi.org/10.1016/j.radonc.2010.03.017.

[21] Imura M, Yamazaki K, Shirato H, Onimaru R, Fujino M, Shimizu S, et al. Insertion and fixation of fiducial markers for setup and tracking of lung tumors in radiotherapy. Int J Radiat Oncol Biol Phys 2005;63:1442-7. https://doi.org/ 10.1016/j.ijrobp.2005.04.024.

[22] Geraghty PR, Kee ST, McFarlane G, Razavi MK, Sze DY, Dake MD. CT-guided transthoracic needle aspiration biopsy of pulmonary nodules: needle size and pneumothorax rate. Radiology 2003;229:475-81. https://doi.org/10.1148/ radiol.2291020499.

[23] Arslan S, Yilmaz A, Bayramgürler B, Uzman O, Nver E, Akkaya E. CT- guided transthoracic fine needle aspiration of pulmonary lesions: accuracy and complications in 294 patients. Med Sci Monit 2002;8:CR493-7.

[24] Coolens C, Bracken J, Driscoll B, Hope A, Jaffray D. Dynamic volume vs respiratory correlated 4DCT for motion assessment in radiation therapy simulation. Med Phys 2012;39:2669-81. https://doi.org/10.1118/1.4704498.

[25] Richter A, Wilbert J, Baier K, Flentje M, Guckenberger M. Feasibility study for markerless tracking of lung tumors in stereotactic body radiotherapy. Int J Radiat Oncol Biol Phys 2010;78:618-27. https://doi.org/10.1016/j.ijrobp.2009.11.028.

[26] Molloy JA, Chan G, Markovic A, McNeeley S, Pfeiffer D, Salter B, et al. Quality assurance of U.S.-guided external beam radiotherapy for prostate cancer: report of AAPM Task Group 154. Med Phys 2011;38:857-71. https://doi.org/10.1118/ 1.3531674 .

[27] Serago CF, Chungbin SJ, Buskirk SJ, Ezzell GA, Collie AC, Vora SA. Initial experience with ultrasound localization for positioning prostate cancer patients for external beam radiotherapy. Int J Radiat Oncol Biol Phys 2002;53:1130-8. https:// doi.org/10.1016/S0360-3016(02)02826-2.

[28] Bell MAL, Sen HT, Iordachita I, Kazanzides P, Wong J. In vivo reproducibility of robotic probe placement for a novel ultrasound-guided radiation therapy system. J Med Imaging 2014;1:025001. https://doi.org/10.1117/1.jmi.1.2.025001.

[29] Fargier-Voiron M, Presles B, Pommier P, Rit S, Munoz A, Liebgott H, et al. Impact of probe pressure variability on prostate localization for ultrasound-based imageguided radiotherapy. Radiother Oncol 2014;111:132-7. https://doi.org/10.1016/ j.radonc.2014.02.008.

[30] Langen KM, Pouliot J, Anezinos C, Aubin M, Gottschalk AR, Hsu IC, et al. Evaluation of ultrasound-based prostate localization for image-guided radiotherapy. Int J Radiat Oncol Biol Phys 2003;57:635-44. https://doi.org/ 10.1016/S0360-3016(03)00633-3.

[31] Orton NP, Jaradat HA, Tomé WA. Clinical assessment of three-dimensional ultrasound prostate localization for external beam radiotherapy. Med Phys 2006; 33:4710-7. https://doi.org/10.1118/1.2388153.

[32] Western C, Hristov D, Schlosser J. Ultrasound imaging in radiation therapy: from interfractional to intrafractional guidance. Cureus 2015;7. https://doi.org/ 10.7759/cureus. 280.

[33] Schlosser J, Salisbury K, Hristov D. Telerobotic system concept for real-time softtissue imaging during radiotherapy beam delivery. Med Phys 2010;37:6357-67. https://doi.org/10.1118/1.3515457.

[34] Schlosser J, Salisbury K, Hristov D. Image-based approach to respiratory gating for liver radiotherapy using a telerobotic ultrasound system. Int J Radiat Oncol 2011; 81:S122. https://doi.org/10.1016/j.ijrobp.2011.06.250.

[35] Sen HT, Bell MAL, Iordachita I, Wong J, Kazanzides P. A cooperatively controlled robot for ultrasound monitoring of radiation therapy. IEEE Int. Conf. Intell. Robot. Syst., vol. 2013, Rep U S; 2013, p. 3071-6. https://doi.org/10.1109/ IROS.2013.6696791.

[36] Hsu A, Miller NR, Evans PM, Bamber JC, Webb S. Feasibility of using ultrasound for real-time tracking during radiotherapy. Med. Phys., vol. 32, John Wiley and Sons Ltd; 2005, p. 1500-12. https://doi.org/10.1118/1.1915934.

[37] O'Shea TP, Bamber JC, Harris EJ. Temporal regularization of ultrasound-based liver motion estimation for image-guided radiation therapy. Med Phys 2016;43: 455-64. https://doi.org/10.1118/1.4938582.

[38] O'Shea T, Bamber J, Fontanarosa D, van der Meer S, Verhaegen F, Harris E. Review of ultrasound image guidance in external beam radiotherapy part II: intra-fraction motion management and novel applications. Phys Med Biol 2016;61:R90-137. https://doi.org/10.1088/0031-9155/61/8/R90.

[39] Fontanarosa D, van der Meer S, Bamber J, Harris E, O'Shea T, Verhaegen F. Review of ultrasound image guidance in external beam radiotherapy: I. Treatment planning and inter-fraction motion management. Phys Med Biol 2015;60:R77-114. https://doi.org/10.1088/0031-9155/60/3/R77. 
[40] van Herk M, McWilliam A, Dubec M, Faivre-Finn C, Choudhury A. Magnetic resonance imaging-guided radiation therapy: a short strengths, weaknesses, opportunities, and threats analysis. Int J Radiat Oncol Biol Phys 2018;101: 1057-60. https://doi.org/10.1016/j.ijrobp.2017.11.009.

[41] Rosenberg SA, Henke LE, Shaverdian N, Mittauer K, Wojcieszynski AP, Hullett CR, et al. A multi-institutional experience of MR-guided liver stereotactic body radiation therapy. Adv Radiat Oncol 2019;4:142-9. https://doi.org/10.1016/j. adro.2018.08.005.

[42] Bayouth JE, Low DA, Zaidi H. MRI-linac systems will replace conventional IGRT systems within 15 years. Med Phys 2019. https://doi.org/10.1002/mp.13657.

[43] Lee W, Chan H, Chan P, Fiorillo T, Fiveland E, Foo T, et al. A magnetic resonance compatible E4D ultrasound probe for motion management of radiation therapy. 2017 IEEE Int. Ultrason. Symp., IEEE; 2017, p. 1-4. https://doi.org/10.1109/ ULTSYM.2017.8092223.

[44] Shepard AJ, Wang B, Foo TKF, Bednarz BP. A block matching based approach with multiple simultaneous templates for the real-time 2D ultrasound tracking of liver vessels. Med Phys 2017;44:5889-900. https://doi.org/10.1002/mp.12574.

[45] Feng M, Balter JM, Normolle D, Adusumilli S, Cao Y, Chenevert TL, et al. Characterization of pancreatic tumor motion using cine MRI: surrogates for tumor position should be used with caution. Int J Radiat Oncol Biol Phys 2009;74: 884-91. https://doi.org/10.1016/j.ijrobp.2009.02.003.

[46] Paganelli C, Seregni M, Fattori G, Summers P, Bellomi M, Baroni G, et al. Magnetic resonance imaging-guided versus surrogate-based motion tracking in liver radiation therapy: a prospective comparative study. Int J Radiat Oncol Biol Phys 2015;91:840-8. https://doi.org/10.1016/j.ijrobp.2014.12.013.

[47] Shimohigashi Y, Toya R, Saito T, Ikeda O, Maruyama M, Yonemura K, et al. Tumor motion changes in stereotactic body radiotherapy for liver tumors: an evaluation based on four-dimensional cone-beam computed tomography and fiducial markers. Radiat Oncol 2017;12:61. https://doi.org/10.1186/s13014-017-0799-7.

[48] Tryggestad E, Flammang A, Han-Oh S, Hales R, Herman J, McNutt T, et al. Respiration-based sorting of dynamic MRI to derive representative 4D-MRI for radiotherapy planning. Med Phys 2013;40. https://doi.org/10.1118/1.4800808.
[49] Cai J, Chang Z, Wang Z, Paul Segars W, Yin FF. Four-dimensional magnetic resonance imaging (4D-MRI) using image-based respiratory surrogate: a feasibility study. Med Phys 2011;38:6384-94. https://doi.org/10.1118/1.3658737.

[50] Liu Y, Yin FF, Chang Z, Czito BG, Palta M, Bashir MR, et al. Investigation of sagittal image acquisition for 4D-MRI with body area as respiratory surrogate. Med Phys 2014;41. https://doi.org/10.1118/1.4894726.

[51] Paganelli C, Summers P, Bellomi M, Baroni G, Riboldi M. Liver 4DMRI: a retrospective image-based sorting method. Med Phys 2015;42:4814-21. https:// doi.org/10.1118/1.4927252.

[52] Wachinger C, Yigitsoy M, Rijkhorst EJ, Navab N. Manifold learning for imagebased breathing gating in ultrasound and MRI. Med Image Anal 2012;16:806-18. https://doi.org/10.1016/j.media.2011.11.008.

[53] von Siebenthal M, Székely G, Gamper U, Boesiger P, Lomax A, Cattin P. 4D MR imaging of respiratory organ motion and its variability. Phys Med Biol 2007;52: 1547-64. https://doi.org/10.1088/0031-9155/52/6/001.

[54] Giger A, Stadelmann M, Preiswerk F, Jud C, De Luca V, Celicanin Z, et al. Ultrasound-driven 4D MRI. Phys Med Biol 2018;63. https://doi.org/10.1088/ 1361-6560/aaca1d.

[55] Cao K, Bednarz B, Smith LS, Foo TKF, Patwardhan KA. Respiration induced fiducial motion tracking in ultrasound using an extended SFA approach. Med. Imaging 2015 Ultrason. Imaging Tomogr., vol. 9419, SPIE; 2015, p. 94190S. https://doi. org/10.1117/12.2082591.

[56] Klüter S. Technical design and concept of a 0.35 T MR-Linac. Clin Transl Radiat Oncol 2019;18:98-101. https://doi.org/10.1016/j.ctro.2019.04.007.

[57] Keiper TD, Tai A, Chen X, Paulson E, Lathuili F, Eriault SB, et al. Feasibility of realtime motion tracking using cine MRI during MR-guided radiation therapy for abdominal targets 2020. https://doi.org/10.1002/mp.14230.

[58] Matrosic CK, Bednarz B, Culberson W. Development and Benchmarking of an Abdominal Phantom for Intrafraction Image Guidance Validation. Phys Med Biol 2020; In Print. 